\title{
Reducing the Read Noise of HAWAII-2RG Detector Systems with Improved Reference Sampling and Subtraction (IRS ${ }^{2}$ )
}

\author{
Bernard J. Rauscher ${ }^{a}$, Richard G. Arendt ${ }^{b}$, D.J. Fixsen ${ }^{c}$, Matthew Lander ${ }^{f}$, Don Lindler ${ }^{d}$, \\ Markus Loose ${ }^{e}$, S. H. Moseley ${ }^{a}$, Donna V. Wilson ${ }^{f}$, and Christos Xenophontos ${ }^{f}$ \\ ${ }^{a}$ Code 665, NASA Goddard Space Flight Center, Greenbelt, MD \\ ${ }^{b}$ CRESST/UMBC/GSFC,Greenbelt, MD \\ ${ }^{c}$ CRESST/UMd/GSFC, Greenbelt, MD \\ ${ }^{d}$ Sigma Space Corporation/GSFC, Greenbelt, MD \\ ${ }^{e}$ Markury Scientific, Inc., 518 Oakhampton Street, Thousand Oaks, CA

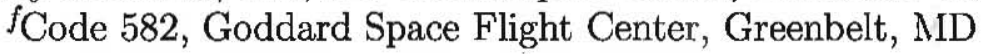

\begin{abstract}
IRS $^{2}$ is a Wiener-optimal approach to using all of the reference information that Teledyne's HAWAII-2RG detector arrays provide. Using a new readout pattern, IRS ${ }^{2}$ regularly interleaves reference pixels with the normal pixels during readout. This differs from conventional clocking, in which the reference pixels are read out infrequently, and only in a few rows and columns around the outside edges of the detector array. During calibration, the data are processed in Fourier space, which is close to the noise's eigenspace. Using IRS ${ }^{2}$, we have reduced the read noise of the James Webb Space Telescope Near Infrared Spectrograph by $15 \%$ compared to conventional readout. We are attempting to achieve further gains by calibrating out recently recognized non-stationary noise that appears at the frame rate.
\end{abstract}

Keywords: HAWAII-2RG, H2RG, SIDECAR, noise, read noise 